\title{
Immunocytochemical localization of claudin-1 in the maturation ameloblasts of rat incisors
}

\author{
Sumio Nishikawa* and Michio Abe \\ Department of Biology, Tsurumi University School of Dental Medicine, Yokohama, Kanagawa, Japan
}

\section{Edited by:}

Hervé Lesot, Université de Strasbourg,

France

\section{Reviewed by:}

Toshio Yamamoto, Okayama University, Japan

Antonius Bronckers, Academic Centre for Dentistry Amsterdam, Netherlands

*Correspondence:

Sumio Nishikawa, Department of

Biology, Tsurumi University School of

Dental Medicine, 2-1-3Tsurumi,

Tsurumi-ku, Yokohama 230-8501,

Japan.

e-mail: nishikawa-s@tsurumi-u.ac.jp
Claudin-1 is a tight junction transmembrane protein. Its localization in the maturation ameloblasts of rat incisors was examined by immunofluorescence microscopy. Distal junction area of ruffle-ended ameloblasts (RA) and the Golgi apparatus of a sub-population of smooth-ended ameloblasts (SA) and RAs stained positive with anti-claudin-1 antibodies. Since it has been shown that ameloblasts repeatedly alternate between an SA and an RA morphology during enamel maturation, the presence of claudin-1 in the Golgi cisterns may indicate the presence of tight junction precursors before transportation to the junctional area.

Keywords: amelogenesis, ruffle-ended ameloblasts, smooth-ended ameloblasts, claudin-1, tight junction, immunofluorescence microscopy

\section{INTRODUCTION}

Mammalian epithelial cells have junctional complexes between cells that are composed of adherens junctions, desmosomes, and tight junctions (Farquhar and Palade, 1963). Tight junctions are responsible for sealing of the intercellular space and for controlling paracellular transport (Tsukita et al., 2008; Lal-Nag and Morin, 2009). Tight junctions are composed of several protein components, among which claudins are essential for their formation and function (Tsukita et al., 2008). In mammals, humans have 23 claudin genes, whereas rats and mice have 24 genes (Lal-Nag and Morin, 2009). Dynamics of tight junction proteins are not fully understood (González-Mariscal et al., 2007; Matter and Balda, 2007; Shen et al., 2008; Steed et al., 2010). Although some dynamics of exogenously expressed claudin-1 and internalization of claudins via small vesicles from tight junction are reported (Sasaki et al., 2003; Matsuda et al., 2004), no information has been given on the recruitment of claudins from cytoplasm to tight junction.

It is known that tooth enamel formation occurs in roughly two stages: the first stage is a secretion stage and the second is a maturation stage. Epithelial ameloblasts are responsible for both stages of enamel formation (Warshawsky and Smith, 1974). Secretion ameloblasts and maturation ameloblasts have tight junctions which have been demonstrated in freeze fracture replica studies (Warshawsky, 1978; Sasaki, 1990). The expression of claudins in ameloblasts has also been reported in immunocytochemical and in situ hybridization studies (João and Arana-Chavez, 2004; Bello et al., 2007; Ohazama and Sharpe, 2007; Inai et al., 2008; Hata et al., 2010). Maturation ameloblasts undergo several modulation cycles, involving two different types of cell morphology, during enamel maturation (Josephsen and Fejerskov, 1977). These two types of ameloblasts are referred to as ruffle-ended ameloblasts (RA) and smooth-ended ameloblasts (SA), respectively. RAs have well-developed tight junctions at the distal junctional area, whereas SAs have moderately developed tight junctions at the proximal junctional area (Sasaki, 1990). ${ }^{45} \mathrm{Ca}$ autoradiography studies have shown that at least calcium ions can pass through the intercellular space of secretory ameloblasts and SAs in a short time, but that these ions pass through the RA layer more slowly (Kawamoto and Shimizu, 1997). Thus, RAs may have tight permeability barriers between cells. Moreover, modulation cycles involve the rapid movement of wave-like bands of RA and SA over the ameloblast layer, that are a result of extensive RA-to-SA or SA-to-RA changes during maturation. Such changes may occur up to 45 times in a mandibular incisor (Josephsen, 1983; Smith et al., 1987). Thus, it is of interest to examine how tight junctions form and break during extensive modulation cycles.

In this study, the localization of claudin-1 in maturation ameloblasts was examined using immunofluorescence microscopy. Unexpectedly, the anti-claudin-1 antibody localized claudin to the Golgi apparatus of a sub-population of SA and RA in addition to the distal junctions of RA. Localization of claudin- 1 in the Golgi apparatus may reflect rapid tight junction turnover during enamel maturation.

\section{MATERIALS AND METHODS}

Wistar rats were used for the present study (Jcl Wistar; Clea Japan, Tokyo, Japan). Twelve 4-week-old rats were sacrificed by decapitation under deep anesthesia with inhalation of diethyl ether or by intraperitoneal injection of sodium pentobarbital (Nembutal; Abbot, North Chicago, IL, USA). Institutional guidelines for animal care were followed for all experimental procedures.

The maxillae and mandibles were dissected and fixed in $4 \%$ paraformaldehyde solution in phosphate-buffered saline (PBS) for $20 \mathrm{~h}$. The maxillae and mandibles were further demineralized with $5 \%$ EDTA, pH 7.2 (adjusted with $\mathrm{NaOH}$ ) at $4^{\circ} \mathrm{C}$ for 3 weeks. The tissues were washed with PBS, were infused with $25 \%$ sucrose in PBS overnight, and were cut into 6- to 9- $\mu \mathrm{m}$-thick cryosections using a cryotome (HM505E; Microm, Walldorf, Germany). 
The cryosections on glass microscope slides were incubated in $1 \%$ bovine serum albumin (BSA) in PBS at RT for $30 \mathrm{~min}$. The sections were then incubated with rabbit anti-claudin-1 antibody (JAY.8; Invitrogen, Camarillo, CA, USA) at a concentration of $12.5 \mu \mathrm{g} / \mathrm{mL}$ in $1 \%$ BSA-PBS overnight at $4^{\circ} \mathrm{C}$. After washing in PBS, the sections were incubated with Alexa 488-conjugated donkey anti-rabbit IgG diluted 1:100, $0.5 \mu \mathrm{g} / \mathrm{mL}$ Hoechst 33342 and rhodamine-phalloidin diluted 1:50 (Molecular Probes, Eugene, OR, USA) at RT for $30 \mathrm{~min}$. Control sections were incubated with normal rabbit immunoglobulin (Dako, Glostrup, Denmark) at a concentration of $10 \mu \mathrm{g} / \mathrm{mL}$ instead of with the primary antibody, and were processed in the same way as described above. Some sections were incubated with mouse monoclonal anti-GM130 antibody (clone 35/GM130, BD Biosciences, CA, USA) at a concentration of $12.5 \mu \mathrm{g} / \mathrm{mL}$ in $1 \%$ BSA-PBS overnight at $4^{\circ} \mathrm{C}$. GM130 is a structural element protein of the Golgi apparatus. The sections were incubated with Alexa 647-conjugated goat antimouse IgG diluted 1:50 at RT for $30 \mathrm{~min}$. Control sections were incubated with normal mouse IgG (Dako) at a concentration of $12.5 \mu \mathrm{g} / \mathrm{mL}$ instead of with the primary antibody, and were processed in the same way as described above. Fluorescence images were obtained using an epifluorescence microscope (AX-80; Olympus, Tokyo, Japan) equipped with a CCD camera (Quantix KAF1401E, Photometrix, Tucson, AZ, USA). Some images were processed using MetaMorph software (Universal imaging, Downington, PA, USA).

\section{RESULTS}

We first examined the localization of claudin in different incisor regions using immunofluorescence. Anti-claudin-1 antibodies did not label ameloblasts in the secretion zone of the incisor. However, in the postsecretory transition zone, the supranuclear region of ameloblasts was labeled by these antibodies in a linear pattern. In the maturation zone, ameloblasts were labeled at the distal end of the zone, along the long axis of the incisor. This labeling was then interrupted but labeling could then be again detected further along this axis (Figure 1). In addition, the supranuclear region of some of the ameloblasts was intensely labeled with these antibodies.

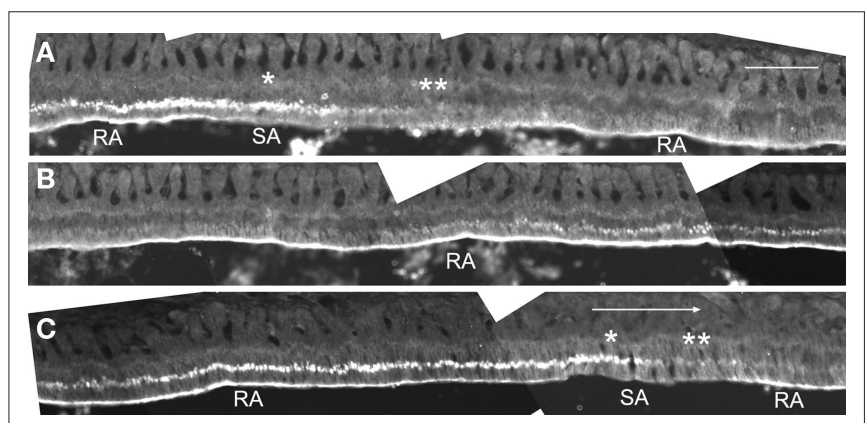

FIGURE 1 | Immunofluorescent localization of claudin-1 at the distal end and at the supranuclear region of maturation ameloblasts. Cryosections of rat mandibles were analyzed for claudin expression using

immunofluorescence. The tissue at the right end of the micrographs in (A) and (B) is continuous with the tissue shown at the left end of the micrographs in (B) and (C), respectively. The large arrow shows incisal direction. Single and double asterisks show regions of the SA bands with and without supranuclear reactivity of claudin-1, respectively. RA, ruffle-ended ameloblast; SA, smooth-ended ameloblast. Bar $=100 \mu \mathrm{m}$.
To determine the characteristics of the maturation ameloblasts with or without distal labeling of anti-claudin-1, the sections were triply stained for claudin-1, F-actin and nuclear DNA using anticlaudin-1, rhodamine-phalloidin, and Hoechst33342 respectively. The distal end of ameloblasts that stained with anti-claudin-1 were co-stained with rhodamine-phalloidin (Figures 2A,C,D,F,G,I), whereas ameloblasts that did not stain with anti-claudin-1 at the distal cell end were not labeled with rhodamine-phalloidin (Figures 2B,E,H). Since F-actin is abundant at the distal end of RAs in which a junctional complex is well developed, but is not expressed at the distal end of SAs, which have poorly developed intercellular junctions at the proximal end (Nishikawa and Josephsen, 1987), the ameloblasts stained at the distal end with anti-claudin-1 are considered to be RAs. No anti-claudin-1 staining was observed at the proximal end of either RAs or SAs.

Some of the maturation ameloblasts also showed positive anticlaudin-1 staining in the supranuclear region. These ameloblasts first appeared as part of an RA band. With progression in an incisal direction these ameloblasts then formed part of an adjacent SA band. During this progression the ameloblasts gradually lost their anti-claudin-1 immunoreactivity in the supranuclear region (Figures 1 and 2A,B). To clarify the nature of the anti-claudin-1positive supranuclear structure, double labeling with anti-Golgi matrix protein GM130 and anti-claudin-1 antibodies was performed. The result showed that GM130 and claudin-1 co-localized in the maturation ameloblasts, but that only GM130 could be detected in the papillary layer cells (Figure 3). To confirm the specificity of anti-claudin- 1 antibody used in this study, perineurium of nerve fibers in incisor periodontal ligaments and molar gingival epithelia were examined, since claudin-1 has localized in perineurium (Pummi et al., 2004) and junctional epithelia of gingiva (Fujita et al., 2010). The result showed the bright labelings in the perineurium surrounding nerve fibers (Figure 4A) and cell periphery of junctional epithelia (Figure 4B). In mandibular incisors the modulation wave of RAs and SAs moves in an incisal direction at a speed of $243 \mu \mathrm{m} / \mathrm{h}$. Thus, each ameloblast on the maturation enamel continuously changes its shape from an RA type to an apically adjacent SA type, and from an SA type to an apically adjacent RA type, when a particular modulation band moves in the incisal direction of the incisor (Figure 5). These changes would explain why, in a particular RA band, a maturation ameloblast reacts positively with anti-claudin- 1 in the distal tight junctions but shows no supranuclear labeling (Figure 5A, nG). Furthermore, they would explain why, in an apically located SA band, maturation ameloblasts initially show no anti-claudin-1 immunoreactivity, but later display immunoreactivity in the supranuclear region (Figure 5B, pG). When the section is viewed from a more apical direction, ameloblasts are observed to change to an RA shape and to develop distal end-labeling of claudin-1 in addition to claudin-1 supranuclear labeling (Figure 5C). This supranuclear labeling is transient and disappears at a later stage (Figure 5A).

The length of SAs in mandibular incisors that showed positive supranuclear labeling of anti-claudin- 1 was $177 \pm 24 \mu \mathrm{m}$ (mean \pm SD, $n=5$ ) (Figure 1). If the modulation wave moves at a speed of $243 \mu \mathrm{m} / \mathrm{h}$ it may take $43.6 \pm 6.0 \mathrm{~min}$ for claudin to move from the Golgi apparatus to the distal junctional plasma membrane. 

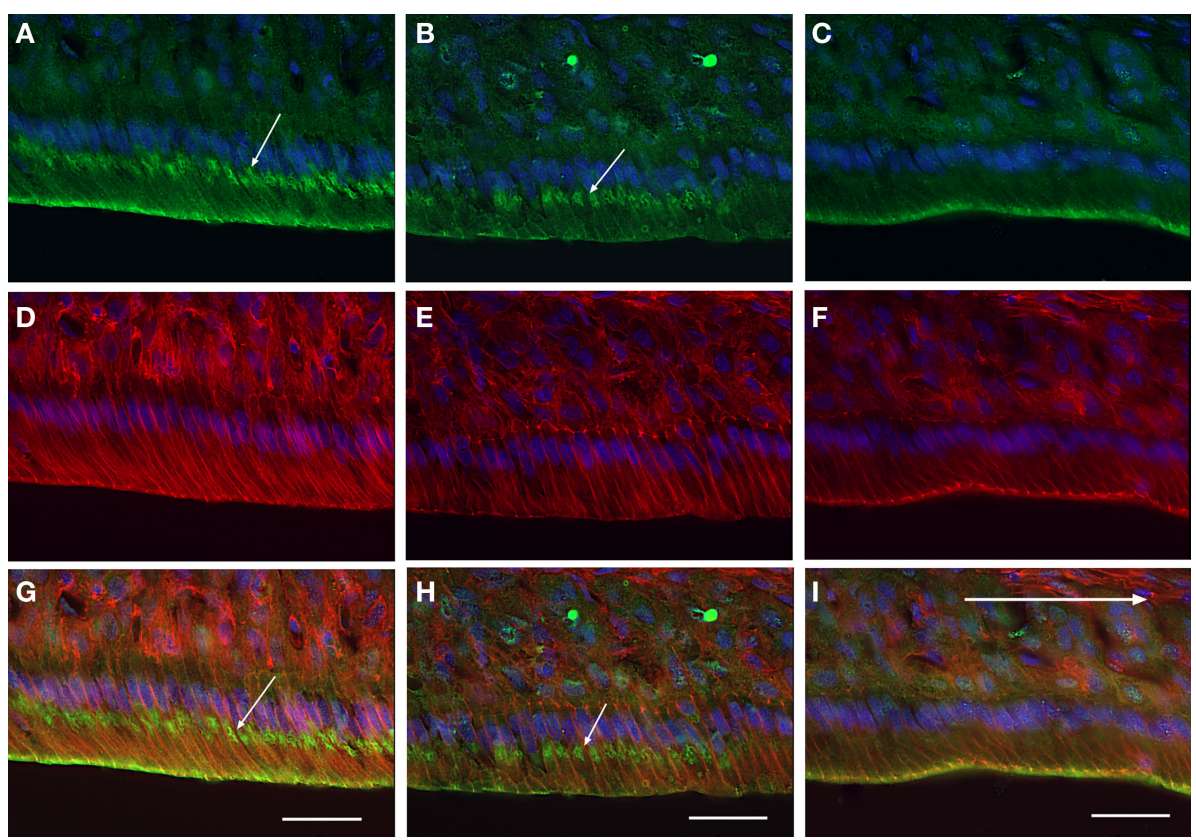

FIGURE 2 | Immunofluorescent localization of claudin-1 in maturation ameloblasts at higher magnification. Cryosections of rat mandibles were triply labeled with anti-claudin-1 [(A-C), green], rhodamine-phalloidin [(D-F), red] and Hoechst 33342 [(A-I), blue], to co-stain claudin, actin and nuclei respectively. (G), (H), and (I) show merged images of $(\mathbf{A}, \mathbf{D}),(\mathbf{B}, \mathbf{E})$, and $(\mathbf{C}, \mathbf{F})$, respectively. The incisal region of an $R A$ band $(\mathbf{A}, \mathbf{D}, \mathbf{G})$, a part of one SA band
$(\mathbf{B}, \mathbf{E}, \mathbf{H})$, and the apical region of another RA band (C,F,I) are shown. The distal end of the ameloblasts of RAs exhibits both anti-claudin-1 and rhodaminephalloidin fluorescence (A,C,D,F,G,I). In addition, small round positive fluorescent foci (small arrows) are visible in the supranuclear cytoplasm of the incisal region of the $R A$ and in some of the $S A$ [arrows in $(\mathbf{A}, \mathbf{B}, \mathbf{G}, \mathbf{H})$ ]. Bars $=30 \mu \mathrm{m}$.
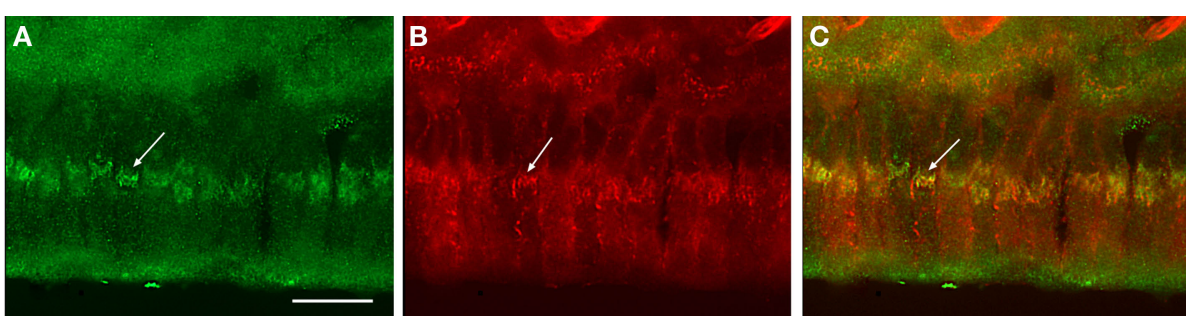

FIGURE 3 | Co-immunofluorescent staining of claudin-1 and a Golgi marker in maturation ameloblasts. Cryosections of rat mandibles were doubly labeled with anti-claudin-1 (A) and anti-GM130 (B). A merged image is shown in
(C). Some anti-claudin-1-positive thread-like clusters co-localize with the Golgi apparatus that is labeled with anti-GM130 in maturation ameloblasts (arrows). Bar $=15 \mu \mathrm{m}$.

\section{DISCUSSION}

In this paper we have further clarified the role of claudin and tight junction formation during cyclic alternation of ameloblasts between an RA and an SA morphology in the process of enamel maturation in rat incisors. We found that claudin-1, which is critical for tight junction formation, is localized in distal junction area in RAs. This finding is consistent with previous data regarding the importance of tight junctions, and of claudins, for enamel formation.

Tight junctional strands have been observed in the proximal and distal junctional complexes of secretion ameloblasts (Warshawsky, 1978) as well as in maturation ameloblasts (Sasaki, 1990). In particular, RAs have well-developed tight junctional strands at their distal end which limit the permeability of the intercellular space, whereas SAs have less developed tight junctional strands
(Sasaki, 1990). Several claudin protein species have been found in tooth bud epithelia and ameloblasts in studies using in situ hybridization probes and antibodies against tight junction proteins (Bello et al., 2007; Ohazama and Sharpe, 2007; Inai et al., 2008; Hata et al., 2010). Of these claudins, claudin-1, -4 , and -7 , and occludin have been detected in ameloblasts by immunocytochemistry, and claudin- 1 and -4 have been localized at the distal end of RAs, but not of SAs, in the maturation zone of incisors (Inai et al., 2008). Although claudin-1 has been detected in presecretory and secretory ameloblasts in addition to maturation ameloblasts (João and Arana-Chavez, 2004; Inai et al., 2008; Hata et al., 2010), in this study ameloblasts anterior to postsecretory transition stage were not labeled by anti-claudin-1. This discrepancy may reflect the different fixation conditions: $4 \%$ paraformaldehyde (PFA) for 


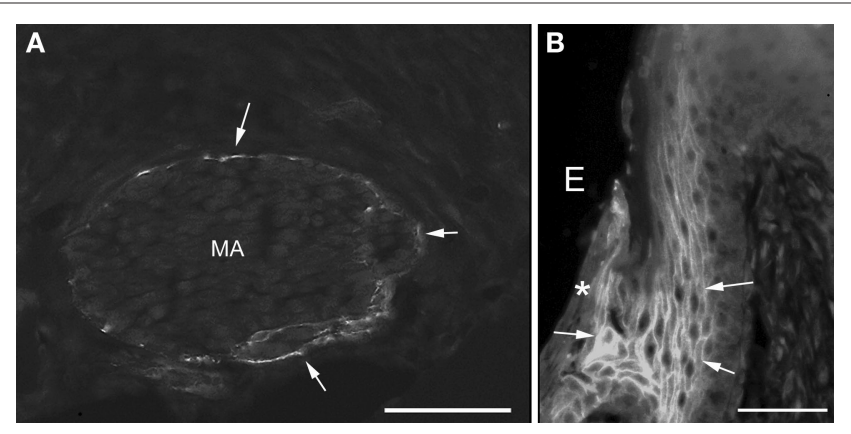

FIGURE 4 | Immunofluorescent staining of claudin-1 in the nerve fibers in incisor periodontal ligaments $(A)$ and gingiva of mandibular third molar (B). The perineurium [arrows in (A)] and the cell periphery of junctional epithelia [arrows in (B)] were labeled with anti-claudin-1. Asterisk shows the surface of junctional epithelia. MA, myelinated axons; $E$, enamel space. Bars $=50 \mu \mathrm{m}$

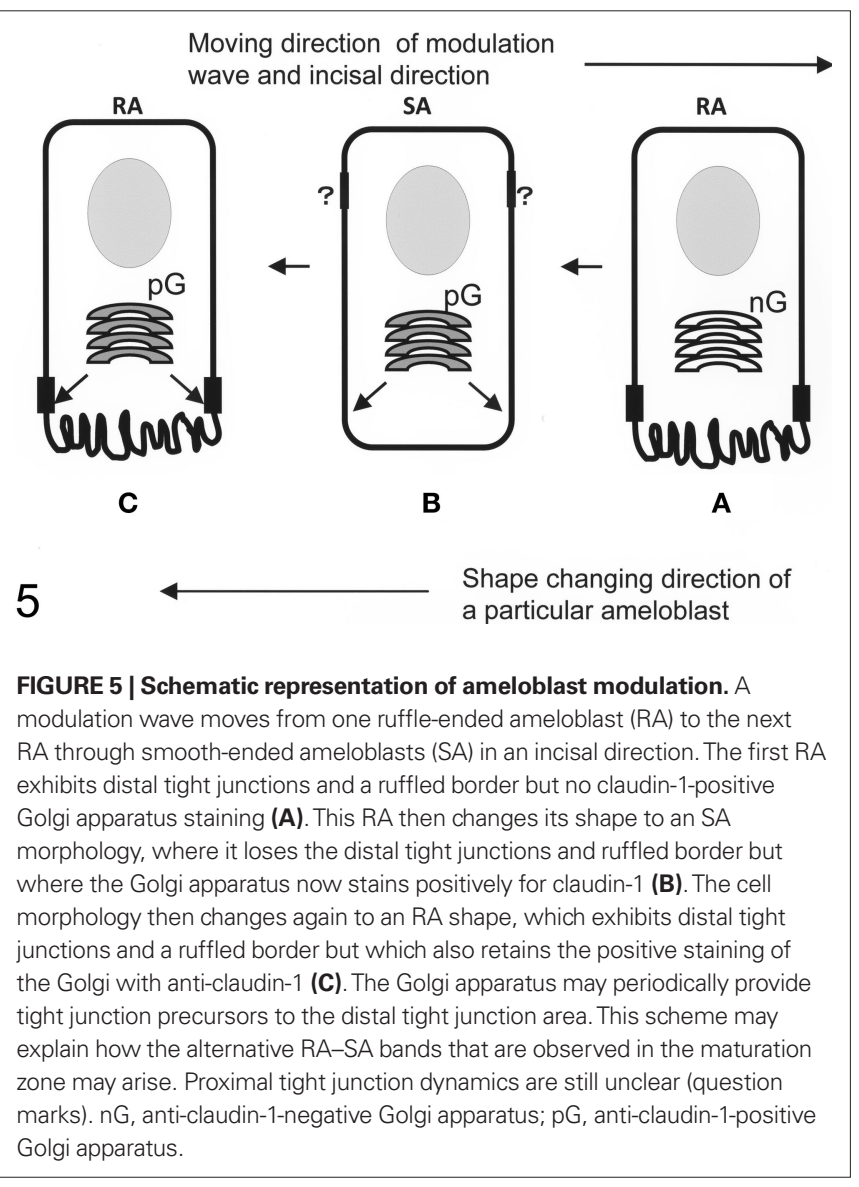

$20 \mathrm{~h}$ in this study versus Zamboni's fixative including 2\% PFA for $4 \mathrm{~h}$ (João and Arana-Chavez, 2004), 1\% PFA for $4 \mathrm{~h}$ (Inai et al., 2008), and cold ethanol for $30 \mathrm{~min}$ and acetone for $1 \mathrm{~min}$ (Hata et al., 2010). Although milder fixation condition may recover the antigenicity of ameloblast claudin- 1 at the earlier stages, the labeling pattern of distal end of maturation ameloblasts observed in this study is consistent with a previous report (Inai et al., 2008). Specificity of the anti-claudin antibody used in this study was confirmed by using positive control sections of perineurium and junctional epithelium. It is noteworthy that, in the present study, claudin-1 was localized in maturation ameloblasts, since epidermal claudin-1, but not claudin-4, plays a role in the tight junction barrier against water loss from mouse skin (Furuse et al., 2002). During enamel maturation, the control of protein and water resorption from enamel and the subsequent increased mineralization of enamel are essential.

Although the ameloblasts themselves are fixed on the enamel surface, they continuously alternate between an RA and an SA morphology, which has been described as modulation bands. This cyclic modulation was reported for the first time by Josephsen (1983). Smith et al. (1987) reported that these modulation bands move in an incisal direction at a rate of $243 \mu \mathrm{m} / \mathrm{h}$ in mandibular incisors and at $188 \mu \mathrm{m} / \mathrm{h}$ in maxillary incisors. By the end of enamel maturation in mandibular incisors, a group of maturation ameloblasts may have undergone 45 RA/SA modulation cycles (Smith et al., 1987), suggesting rapid and extensive turnover of tight junctions. To understand tight junction turnover in maturation ameloblasts, we examined claudin-1 localization in RAs and SAs using immunocytochemistry. The results showed that claudin-1 localized at the distal end of the RAs but not the SAs. RAs and SAs were distinguished by F-actin localization after double labeling with rhodamine-phalloidin and anti-claudin-1, based on a previous study that F-actin is abundant in the distal end of RAs but not in the distal end of SAs (Nishikawa and Josephsen, 1987). These data suggest the model that an SA might convert to an RA by first forming a claudin-1-positive tight junction precursor in the Golgi apparatus. Although the Golgi-derived vesicular precursor of tight junction has not been described, vesicular transport of tight junction protein has been proposed (Matsuda et al., 2004; Steed et al., 2010). This precursor might then move to the distal junction area, at which point the SA becomes an RA that forms tight junctions and extensive membrane ruffles and in which both the Golgi apparatus and the distal cell end stain positively with anti-claudin-1 antibodies. However, further investigation is required to confirm the presence, and nature, of tight junction precursors in the Golgi area. In support of this model, we found that ameloblasts in the apical part of the RA band, where RAs are changing back into SAs, had lost anti-claudin-1 immunoreactivity of the Golgi apparatus, and only their distal end was reactive to anti-claudin-1 (Figures 1 and 2). Ultimately, the anti-claudin-1-positive tight junction may be lost at the distal end of these ameloblasts and instead the cells may make proximal junctional complexes, thereby turning into an SA. In this SA, the tight junction precursor may then again start to form in the Golgi, thereby beginning a new modulation cycle. By measurement of the length of the SA band that displayed supranuclear anti-claudin-1 labeling, the time it would take for the tight junction precursor structure to move from the Golgi apparatus to the distal junctional plasma membrane was calculated as $43.6 \pm 6.0 \mathrm{~min}$. This time is consistent with the dynamic properties of the tight junction strand network which has been shown to reorganize within several minutes in L cells in which claudin-1 was exogenously expressed (Sasaki et al., 2003). However, a second study indicated that individual tight junction strands are relatively stable after photobleaching of a section of a fluorescent tight junction strand 
(Sasaki et al., 2003). Although more studies are clearly needed, it was shown in this study that the rapid alteration of the tight junction of ameloblasts during enamel maturation was accompanied by Golgi complex activities, which imply the involvement of the formation and destruction of tight junction membrane-protein complexes during SA/RA modulation cycles.

\section{REFERENCES}

Bello, I. O., Soini, Y., Slootweg, P. J., and Salo, T. (2007). Claudins 1, 4, 5, 7 and occludin in ameloblastomas and developing human teeth. J. Oral Pathol. Med. 36, 48-54.

Farquhar, M. G., and Palade, G.E. (1963). Junctional complexes in various epithelia. J. Cell Biol. 17, 375-412.

Fujita, T., Hayashida, K., Shiba, H, Kishimoto, A., Matsuda, S., Takeda, K., Kawaguchi, K., and Kurihara, H. (2010). The expressions of claudin-1 and E-cadherin in junctional epithelium. J. Periodont. Res. 45, 579-582.

Furuse, M., Hata, M., Furuse, K., Yoshida, Y., Haratake, A., Sugitani, Y., Noda, T., Kubo, A., and Tsukita, S. (2002). Claudin-based tight junctions are crucial for the mammalian epidermal barrier: a lesson from claudin-1-deficient mice. J. Cell Biol. 156, 1099-1111.

González-Mariscal, L., Lechuga, S., and Garay, E. (2007). Role of tight junctions in cell proliferation and cancer. Prog. Histochem. Cytochem. 42, 1-57.

Hata, M., Kawamoto, T., Kawai, M., and Yamamoto, T. (2010). Differential expression patterns of the tight junction-associated proteins occludin and claudins in secretory and mature ameloblasts in mouse incisor. Med. Mol. Morphol. 43, 102-106.

Inai, T., Sengoku, A., Hirose, E., Iida, H., and Shibata, Y. (2008). Differential expression of the tight junction proteins, claudin-1, claudin-4, occluding, ZO-1, and PAR3, in the ameloblasts of rat upper incisors. Anat. Rec.291, 577-585.
João, S. M. A., and Arana-Chavez, V. E. (2004). Tight junctions in differentiating ameloblasts and odontoblasts differentially express ZO-1, occluding, and claudin-1 in early odontogenesis of rat molars. Anat. Rec. 277A, 338-343.

Josephsen, K. (1983). Indirect visualization of ameloblast modulation in the rat incisor using calcium-binding compounds. Scand. J. Dent. Res. 91, 76-78.

Josephsen, K., and Fejerskov, O. (1977). Ameloblast modulation in the maturuation zone of the rat incisor enamel organ. A light and electron microscopic study. J. Anat. 124, 45-70.

Kawamoto, T., and Shimizu, M. (1997). Pathway and speed of calcium movement from blood to mineralizing enamel. J. Histochem. Cytochem. 45, 213-230.

Lal-Nag, M., and Morin, P. J. (2009). The claudins. Genome Biol. 10, 235.

Matsuda, M., Kubo, A., Furuse, M., and Tsukita, S. (2004). A peculiar internalization of claudins, tight junction-specific adhesion molecules, during the intercellular movement of epithelial cells. J. Cell Sci. 117, 1247-1257.

Matter, K., and Balda, M. S. (2007). Epithelial tight junctions, gene expression and nucleo-junctional interplay. J. Cell Sci. 120, 1505-1511.

Nishikawa, S., and Josephsen, K. (1987). Cyclic localization of actin and its relationship to junctional complexes in maturation ameloblasts of the rat incisor. Anat. Rec. 219, 21-31.

\section{ACKNOWLEDGMENTS}

The authors thank Y. Ishikawa, K. Osawa, and M. Kogiku for their technical assistance. This work was supported in part by a Grant for Supporting Project for Strategic Research of Nihon University School of Dentistry at Matsudo by the Ministry of Education, Culture, Sports, Science and Technology, 2008-2012.

Ohazama, A., and Sharpe, P. T. (2007) Expression of claudins in murine tooth development. Dev. Dyn. 236, 290-294.

Pummi, K. P., Heape, A. M., Grénman, R. A., Peltonen, J. T. K., and Peltonen, S. A. (2004). Tight junction proteins $\mathrm{ZO}-1$, occludin, and claudins in developing and adult human perineurium. J. Histochem. Cytochem. 52 1037-1046.

Sasaki, H., Matsui, C., Furuse, K., MimoriKiyose, Y., Furuse, M., and Tsukita, S. (2003). Dynamic behavior of paired claudin strands within apposing plasma membranes. Proc. Natl. Acad. Sci. U.S.A. 100, 3971-3976.

Sasaki, T. (1990). Cell biology of tooth enamel formation. Monogr. Oral Sci. 14, 1-199.

Shen, L., Weber, C. R., and Turner, J. R. (2008). The tight junction protein complex undergoes rapid and continuous molecular remodeling at steady state. J. Cell Biol. 181, 683-695.

Smith, C. E., McKee, M. D., and Nanci, A. (1987). Cyclic induction and rapid movement of sequential waves of new smooth-ended ameloblast modulation bands in rat incisors as visualized by polychrome fluorescent labeling ad GBHA-staining of maturing enamel. Adv. Dent. Res. 1, 162-175.

Steed, E., Balda, M. S., and Matter, K. (2010). Dynamics and functions of tight junctions. Trends Cell Biol. 20, 142-149.

Tsukita, S., Yamazaki, Y., Katsuno, T., Tamura, A., and Tsukita, S. (2008).
Tight junction-based epithelial microenvironment and cell proliferation. Oncogene 27, 6930-6938.

Warshawsky, H. (1978). A freeze-fracture study of the topographic relationship between inner enamel-secretory ameloblasts in the rat incisor. Am. J. Anat. 152, 153-208.

Warshawsky, H., and Smith, C. E. (1974). Morphological classification of rat incisor ameloblasts. Anat. Rec. 179, 423-446.

Conflict of Interest Statement: The authors declare that the research was conducted in the absence of any commercial or financial relationships that could be construed as a potential conflict of interest.

Received: 25 August 2010; accepted: 26 October 2010; published online: 16 November 2010.

Citation: Nishikawa S and Abe M (2010) Immunocytochemical localization of claudin-1 in the maturation ameloblasts of rat incisors. Front. Physio. 1:150. doi: 10.3389/ fphys.2010.00150

This article was submitted to Frontiers in Craniofacial Biology, a specialty of Frontiers in Physiology.

Copyright $\odot 2010$ Nishikawa and Abe. This is an open-access article subject to an exclusive license agreement between the authors and the Frontiers Research Foundation, which permits unrestricted use, distribution, and reproduction in any medium, provided the original authors and source are credited. 